\title{
Efecto antihipertensivo y dosis letal 50 del jugo del fruto y del extracto etanólico de las hojas de Passiflora edulis (maracuyá), en ratas
}

\author{
Juan Rojas ${ }^{1}$, Sergio Ronceros ${ }^{1}$, Robert Palomino ${ }^{1}$, Gloria Tomás ${ }^{2}$, Julio Chenguayen ${ }^{3}$
}

\begin{abstract}
Resumen
Objetivos: Determinar el efecto antihipertensivo y la dosis letal (DL) 50 del jugo del frutoyel extracto etanólico de las hojas de Passiflora edulis en ratas. Diseño: Estudio prospectivo, analíticoexperimental, preclínico in vivo. Lugar: Facultades de Medicina Humana, de Química e Ingeniería Química y de Farmacia y Bioquímica, Universidad Nacional Mayor de San Marcos. Material biológico: Ratas. Intervenciones: Para el efecto antihipertensivo se utilizó 4 grupos de ratas $(n=6)$. Después de una medición basal, se indujo hipertensión arterial con L-NAME $50 \mathrm{mg} / \mathrm{kg} /$ día VOy transcurridos dos días se inició el tratamiento con agua destilada, extracto etanólico $500 \mathrm{mg} / \mathrm{kg} /$ día VO, jugo del fruto $500 \mathrm{mg} / \mathrm{kg} /$ día v.o, captopril $100 \mathrm{mg} / \mathrm{kg} /$ día VO, respectivamente. Se continuó midiendo la presión arterial en los días 3, 4, 7 y 10. Para determinar la DL50, se utilizó 12 grupos de ratones $(n=10)$; se aplicó 7 niveles de dosis de extracto etanólico y 5 niveles para el jugo, por única vez. Principales medidas de resultados: Disminución de la presión arterial. Resultados: El extracto etanólico y el jugo del fruto disminuyeron la presión arterial sistólica (PAS) desde el primer día de tratamiento: 157,2 $\pm 2,3$ y 156,2 $\pm 2,1 \mathrm{mmHg}$ comparado con 167,7 \pm 1,7 mm Hg del grupo L-NAME $(p<0,005$ y $<<0,001)$, respectivamente; la presión arterial diastólica $(P A D)$ también disminuyó significativamente a partir del primer día. Las DL50 para el extracto etanólico yjugo del fruto fueron 10687 y $21471 \mathrm{mg} / \mathrm{kg}$, respectivamente. Conclusiones: El extracto etanólico de las hojas y el jugo del fruto de Passiflora edulis fueron efectivos en disminuir la presión arterial en ratas hipertensas, que no presentaron toxicidad aguda oral.
\end{abstract}

Palabrasclave Passifloraedulis; hipertensión; investigación.

Antihypertensive effect and lethal dose 50 of Passiflora edulis leaves (maracuya) fruit juice and ethanolic extract in rats

\section{Abstract}

Objetives: To determine the antihypertensive effect and lethal dose (LD) 50 of the fruit juice and ethanolic extract from Passiflora edulis leaves in rats. Design: Prospective, analytic-experimental, preclinical in vivo study. Setting: Faculties of Medicine, Chemistry and Chemical Engineering and Pharmacy and Biochemistry, San Marcos National University. Biologic material: Rats. Interventions: Four groups of rats $(n=6)$ were used to determine the antihypertensive effect. After basal determination, arterial

\footnotetext{
Facultad de Medicina, UNMSM. Lima, Perú.

Facultad Química e Ingeniería Química, UNMSM. Lima, Perú.

Facultad de Farmacia y Bioquímica, UNMSM. Lima, Perú.
}

hypertension was induced using L-NAME $50 \mathrm{mg} / \mathrm{kd} / \mathrm{d}$ VO and two days later the treatment was initiated with distilled water, ethanolic extract (500 mg/kg/d) VO, fruitjuice $500 \mathrm{mg} / \mathrm{kg} / \mathrm{d} V O$, captopril $100 \mathrm{mg} / \mathrm{kg} / \mathrm{d} \mathrm{VO}$, respectively. Arterial pressure was measured on days 3, 4, 7 and 10. For determining LD50, 12 groups of mice ( $n=10$ ) were used with 7 different dose levels of ethanolic extract, and 5 levels for the one dose juice. Main outcome measures: Blood pressure decrease. Results: The ethanolic extract and the fruitjuice decreased the systolic blood pressure (SBP) starting the first day of treatment: 157,2 $\pm 2,3$ and 156,2 $\pm 2,1 \mathrm{mmHg}$ compared to $167,7 \pm 1,7 \mathrm{mmHg}$ of the L-NAME group ( $p<0,005$ yp $<0,001$ ), respectively. Also the diastolic blood pressure (DBP) significantly decreased starting the first day of treatment. LD50 for the ethanolic extract and juice fruit were 10687 and 21471 $\mathrm{mmHg}$, respectively. Conclusions: Ethanolic extract of the leaves and the fruit juice of Passiflora edulis were effective to decrease the arterial blood pressure in hypertensive rats, without oral acute toxicity.

Key words: Passiflora edulis; hypertension; research. 


\section{INTRODUCCIÓN}

El género Passiflora comprende cerca de 500 especies y es el más grande en la familia Passifloraceae $\left({ }^{1}\right)$. Passiflora edulis Sims es una planta originaria de la amazonía brasileña, conocida con el nombre común de maracuyá, parchita, calala, maracujá, yellow passion-fruit $\left(^{2}\right)$. La palabra maracuyá proviene del portugués-brasileño maracuya, de origen indígena, que significa 'comida preparada en Totuma' $\left({ }^{3}\right)$.

Passiflora edulis es una especie cultivada ampliamente en países tropicales y subtropicales; existen dos variedades: Passiflora edulis Sims var. flavicarpa, cuyos frutos son amarillos, crece desde el nivel del mar hasta $1000 \mathrm{msnm}$; y, Passiflora edulis Sims var. purpúrea, con frutos color púrpura y que se adapta a zonas altas por encima de $1200 \mathrm{msnm}\left({ }^{4}\right)$.

El maracuyá es una planta leñosa perenne, de hábito trepador y de rápido desarro110 , que puede alcanzar hasta $10 \mathrm{~m}$ de largo; las hojas son simples, alternas, con estípulas y un zarcillo en la axila, con márgenes aserrados; las flores son solitarias y axilares, fragantes y vistosas; el fruto es una baya esférica, globosa o elipsoide, que mide hasta $10 \mathrm{~cm}$ de diámetro y pesa hasta $190 \mathrm{~g}$, de color amarillo o purpúreo, con una pulpa muy aromática $\left({ }^{5}\right)$.

La información etnofarmacológica revela que Passiflora edulis Sims ha sido utilizada en medicina tradicional en diversas partes del mundo. En la India, las hojas frescas de esta planta son hervidas en pequeña cantidad de agua y el extracto es bebido para el tratamiento de disentería e hipertensión, y los frutos son comidos para el alivio de la constipación $\left({ }^{6}\right)$. En sudamérica, se bebe la infusión de hojas y flores como sedante, la infusión de las partes aéreas se utiliza en el tratamiento de tétanos, epilepsia, insomnio e hipertensión $\left({ }^{7}\right)$; además, es indicada como relajante muscular $\left(^{8}\right)$, diurético, para tratar dolores estomacales, tumores intestinales y fiebre $\left({ }^{9}\right)$.

El estudio fitoquímico de Passiflora edulis Sims (Passifloraceae) demuestra la presencia de glucósidos, entre ellos passiflorina $\left({ }^{10}\right)$, glucósidos flavonoides: luteolina-6-Cchinovóside $\left({ }^{11}\right)$, glucósidos cianogénicos $\left({ }^{12,13}\right)$; alcaloides: harman $\left({ }^{14}\right)$; triterpenos y saponinas $\left({ }^{15}\right)$; fenoles, carotenoides, antocianinas, ácido L-ascórbico, $\gamma$-lactonas, ésteres, aceites volátiles, eugenol, aminoácidos, carbohidratos y minerales ${ }^{1}$ ).

A través de las investigaciones farmacológicas realizadas, el extracto de las hojas de Passiflora edulis demostró ser depresor del sistema nervioso central (SNC), sugiriendo una acción calmante tipo tranquilizante mayor $\left({ }^{16}\right)$ y ansiolítica $\left({ }^{17}\right)$. Por otra parte, el extracto acuoso del fruto inhibió las enzimas que tienen actividad de endopeptidasas dependientes de zinc, las metaloproteinasas de matriz extracelular MMP-2 y MMP-9, involucradas en la invasión tumoral, metástasis y angiogénesis $\left({ }^{18}\right)$.

La búsqueda de fuentes terapéuticas en el reino vegetal constituye una alternativa viable. Passiflora edulis (maracuyá) es un recurso natural abundante en el Perú y fácilmente cultivable en costa y selva. No existe al presente ningún estudio preclínico ni clínico de Passiflora edulis, en relación al efecto antihipertensivo de esta planta o de algún componente aislado de ella, ya que la mayoría de investigaciones de esta especie están referidos a su efecto hipnótico y sedante.

Los objetivos del presente estudio fueron determinar el efecto antihipertensivo y la dosis letal 50 del jugo del fruto y el extracto etanólico de las hojas de Passiflora edulis en un modelo experimental con ratas hipertensas inducidas por LNAME. 


\section{MATERIALES Y MÉTODOS}

El estudio tuvo un diseño de investigación básica, prospectiva, analítica-experimental, preclínica in vivo.

Las hojas y el fruto maduro de maracuyá fueron colectados en el mes de marzo de 2004, en el Distrito de Moche, Provincia de Trujillo, Departamento de la Libertad, Perú. La planta fue identificada en el $\mathrm{Mu}-$ seo de Historia Natural de la Universidad Nacional Mayor de San Marcos, como Passiflora edulis Sims variedad. Flavicarpa.

Las hojas fueron secadas a $38{ }^{\circ} \mathrm{C}$, en un horno con aire circulante. El material seco y pulverizado fue macerado con etanol al $96 \%$, durante 72 horas, a temperatura ambiente. Se filtró y concentró a sequedad en horno con aire circulante, a $38{ }^{\circ} \mathrm{C}$. El residuo seco fue conservado a $4{ }^{\circ} \mathrm{C}$. El jugo de los frutos maduros fue colado, para separarlo de las semillas, filtrado y finalmente liofilizado y conservado hasta su uso.

Para determinar el efecto antihipertensivo, se utilizó 24 ratas macho raza Holtzmann, con peso de $220 \pm 20 \mathrm{~g}$, adquiridas del Instituto Nacional de Salud. Los animales fueron distribuidos al azar en 4 grupos $(n=6)$. Después de una semana de aclimatación, se procedió a medir la presión arterial (basal, D0), con el aparato LE 5002 Letica, el cual está equipado con un microprocesador específicamente diseñado para realizar mediciones indirectas de la presión sanguínea en la cola de animales de experimentación. Enseguida, se indujo hipertensión arterial en todos los grupos, mediante la administración de L-NAME $\left(\mathrm{N}_{\mathrm{G}}\right.$-nitro-L-arginina metil éster) $\left(\right.$ Sigma $\left.^{\circledR}\right)$, en dosis de $50 \mathrm{mg} / \mathrm{kg}$ $1 \mathrm{vez} /$ día VO, durante todo el experimento (método de Pereira y col., 2004) ${ }^{\left({ }^{19}\right)}$. Después de dos días (D2), se inició el tratamiento, de la siguiente manera: primer grupo, agua destilada; segundo grupo, extracto etanólico de las hojas de Passiflora edulis por vía oral, en dosis de $500 \mathrm{mg} / \mathrm{kg} /$ día; tercer grupo, liofilizado del jugo del fruto de Passiflora edulis $500 \mathrm{mg} / \mathrm{kg} / \mathrm{día} \mathrm{VO}$; cuarto grupo, captopril $100 \mathrm{mg} / \mathrm{kg} /$ día VO. El tratamiento prosiguió hasta el final del experimento. La presión arterial se continuó midiendo en los días 3, 4, 7 y 10 (D3, D4, D7 y D10), respectivamente. Se registró los datos de presión arterial sistólica (PAS), presión arterial diastólica (PAD) y presión arterial media (PAM). Las mediciones fueron realizadas diariamente, siempre en el mismo horario y, antes de aplicar el tratamiento, los animales fueron sometidos a un proceso previo de vasodilatación, mediante calentamiento a $32{ }^{\circ} \mathrm{C}$ durante 30 minutos; cada medición diaria fue el resultado del promedio de cinco lecturas realizadas a cada animal.

La dosis letal 50 fue determinada por el método de Vega y Carrillo, $1997\left({ }^{20}\right)$. Se utilizó 120 ratones albinos, que fueron distribuidos aleatoriamente en 12 grupos ( $=10$ ). Después de una semana de aclimatación, previo ayuno de 4 horas, se administró por vía oral, a los primeros 7 grupos, el extracto etanólico de las hojas de Passiflora edulis, en dosis única de 2500 , 5 000, 7 500, 10 000, 12500,15000 y 17500 $\mathrm{mg} / \mathrm{kg}$; los restantes 5 grupos recibieron liofilizado del jugo del fruto, en dosis única de $2500,5000,10000,15000$ y 20000 $\mathrm{mg} / \mathrm{kg}$. Los animales fueron observados constantemente durante las primeras 24 horas y diariamente durante un periodo de 14 días, registrando cualquier signo tóxico. Al finalizar este periodo, se procedió al sacrificio por tracción de la nuca, para hacer la autopsia; se realizó examen macroscópico de órganos y se determinó su peso relativo.

Los resultados obtenidos de las mediciones de presión arterial fueron expresados como media \pm error estándar de la media. Se aplicó el análisis de varianza de una vía y una prueba post hoc LSD, para determinar la diferencia estadística entre los grupos, con un nivel de significancia de $p<0,05$. El valor 
de la dosis letal 50 fue estimado mediante el método estadístico de los Probits. Se usó el software SPSS 11,0 de Microsoft.

\section{RESULTADOS}

La presión arterial se incrementó rápidamente, por efecto del tratamiento con LNAME, desde 129,7 $\pm 1,4 \mathrm{mmHg}$ (D0) hasta $162,7 \pm 1,8 \mathrm{mmHg}$ (D2), en el grupo L-NAME. Un efecto similar se observó en los demás grupos, por lo que marcó el inicio del tratamiento con la muestra. El extracto etanólico de las hojas de Passiflora edulis disminuyó significativamente la presión arterial sistólica (PAS) desde el primer

Tabla 1. Presión arterial (mmHg) en ratas hipertensas por L-NAME, tratadas con Passiflora edulis.

\begin{tabular}{lcccc}
\hline Grupo & Día & PAS & PAD & PAM \\
\hline L-Name & D0 & $129,7 \pm 1,4$ & $80,0 \pm 1,5$ & $96,2 \pm 1,0$ \\
& D2 & $162,7 \pm 1,8$ & $115,5 \pm 5,1$ & $131,0 \pm 3,6$ \\
& D3 & $167,7 \pm 1,7$ & $116,3 \pm 2,9$ & $133,0 \pm 1,9$ \\
& D4 & $169,2 \pm 2,0$ & $123,0 \pm 2,8$ & $137,8 \pm 2,3$ \\
& D7 & $174,7 \pm 3,4$ & $121,5 \pm 5,4$ & $138,8 \pm 4,5$ \\
Hojas & D10 & $179,7 \pm 1,6$ & $136,2 \pm 3,2$ & $150,3 \pm 2,3$ \\
& D0 & $125,7 \pm 2,0$ & $78,3 \pm 3,9$ & $93,8 \pm 2,7$ \\
& D2 & $165,5 \pm 1,9$ & $109,5 \pm 3,6$ & $127,8 \pm 2,9^{\dagger}$ \\
& D3 & $157,2 \pm 2,3^{\dagger}$ & $105,5 \pm 2,1^{*}$ & $122,3 \pm 1,9^{\dagger}$ \\
& D4 & $154,2 \pm 2,0^{\dagger}$ & $108,0 \pm 4,9^{*}$ & $122,8 \pm 3,6^{\dagger}$ \\
& D7 & $145,8 \pm 3,7^{\dagger}$ & $93,2 \pm 3,7$ & $110,2 \pm 3,6^{\dagger}$ \\
& D10 & $154,3 \pm 2,4^{\dagger}$ & $110,7 \pm 4,2$ & $125,0 \pm 3,6^{\dagger}$ \\
& D0 & $121,5 \pm 2,9$ & $74,3 \pm 3,0$ & $89,7 \pm 2,8$ \\
& D2 & $163,0 \pm 1,8$ & $114,0 \pm 6,0$ & $130,2 \pm 4,4$ \\
& D3 & $156,2 \pm 2,1^{\dagger}$ & $104,0 \pm 3,8^{*}$ & $120,8 \pm 2,6^{\dagger}$ \\
& D4 & $149,8 \pm 2,9^{\dagger}$ & $107,0 \pm 3,0^{*}$ & $120,8 \pm 2,8^{\dagger}$ \\
& D7 & $145,3 \pm 3,1^{\dagger}$ & $96,5 \pm 4,5$ & $112,3 \pm 3,8^{\dagger}$ \\
& D10 & $149,2 \pm 2,1^{\dagger}$ & $109,8 \pm 3,2$ & $122,5 \pm 2,5^{\dagger}$ \\
Captopril & D0 & $125,3 \pm 1,5$ & $78,3 \pm 3,0$ & $93,7 \pm 0,9$ \\
& D2 & $166,2 \pm 2,3$ & $129,5 \pm 4,9$ & $141,5 \pm 3,7$ \\
& D3 & $139,8 \pm 2,4$ & $97,0 \pm 4,6$ & $110,7 \pm 3,5$ \\
& D4 & $135,5 \pm 1,9$ & $94,7 \pm 5,4$ & $108,0 \pm 3,8$ \\
& D7 & $137,3 \pm 1,3$ & $90,0 \pm 4,0$ & $105,5 \pm 2,7$ \\
& D10 & $132,2 \pm 2,3$ & $80,7 \pm 3,1$ & $97,5 \pm 2,2$ \\
\hline & & & &
\end{tabular}

Datos son expresados como media aritmética \pm error estándar $P A S=$ Presión arterial sistólica; $P A D=$ Presión arterial diastólica; $P A M=$ Presión arterial media ${ }^{*} p<0,05$ vs. grupo L-NAME ${ }^{\dagger} p<0,005$ vs. grupo L-NAME día de tratamiento (D3), observándose una reducción hasta $157,2 \pm 2,3 \mathrm{mmHg}$ comparado con $167,7 \pm 1,7 \mathrm{mmHg}$ del grupo L-NAME $(p<0,005)$. El efecto reductor de la PAS prosiguió hasta $145 \pm 3,7 \mathrm{mmHg}$ (D7) y al final del experimento (D10) fue $154,3 \pm 2,4 \mathrm{mmHg}$ vs $179,7 \pm 1,6 \mathrm{mmHg}$ del grupo L-NAME $(p<0,001)$. La presión arterial diastólica (PAD) también disminuyó significativamente por este tratamiento, a partir del primer día (D3), donde se registró $105,5 \pm 2,1 \mathrm{mmHg}$ vs. 116,3 $\pm 2,9 \mathrm{mmHg}$ del grupo L-NAME $(p<$ $0,05)$ (Tabla 1).

El jugo del fruto de Passiflora edulis, desde el primer día de tratamiento (D3) disminuyó significativamente la PAS hasta $156,2 \pm 2,1 \mathrm{mmHg}$ vs. $167,7 \pm 1,7 \mathrm{mmHg}$ del grupo L-NAME $(p<0.001)$ y la PAD hasta $104,0 \pm 3,8 \mathrm{mmHg}$ vs. $116,3 \pm 2,9$ mmHg del control L-NAME $(p<0,05)$. Tanto la PAS como la PAD continuaron disminuyendo en el día 7 (D7), hasta 145,3 \pm $3,1 / 96,5 \pm 4,5 \mathrm{mmHg}$; al final del experimento (D10) fue 149,2 $\pm 2,1 / 109,8 \pm 3,2$ mmHg comparado con $179,7 \pm 1,6 / 136,2$ $\pm 3,2 \mathrm{mmHg}$ del control L-NAME $(p<$ 0,001) (Figura 1).

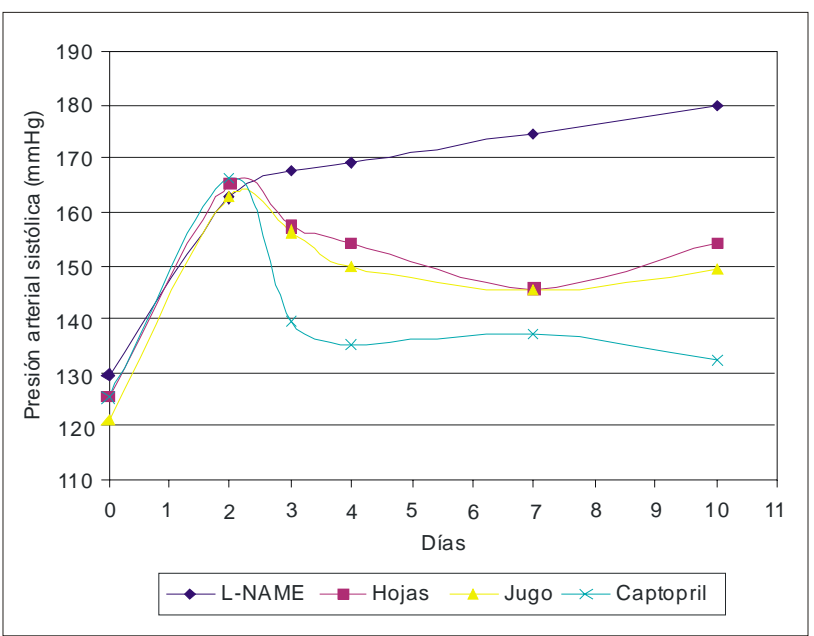

Figura 1. Efecto del tratamiento con Passiflora edulis sobre la presión arterial sistólica, en ratas hipertensas. 
En la determinación de la dosis letal 50, el signo que con mayor frecuencia se observó, en relación directa con la dosis, fue sedación, que en algunos casos desapareció en las primeras 24 horas después de la administración y en otros casos produjo la muerte del animal. La mortalidad obtenida por cada dosis de ensayo, tanto para el extracto de las hojas como para el jugo de Passiflora edulis se muestra en la Tabla 2. La dosis letal 50 estimada para el extracto etanólico de las hojas fue $10687 \mathrm{mg} / \mathrm{kg}$, con límites de confianza del $95 \%$ de 9243 a $12203 \mathrm{mg} / \mathrm{kg}$, mientras que para el jugo del fruto fue $21471 \mathrm{mg} / \mathrm{kg}$, con límites de confianza del $95 \%$ de 17368 a 42769 , tal como se muestra en la Tabla 3 .

Tabla 2. Porcentaje de mortalidad obtenido con el extracto etanólico de las hojas y el jugo de Passiflora edulis.

\begin{tabular}{lrccc}
\hline Tratamiento & Dosis & $\mathrm{n}$ & $\mathrm{N}^{\mathrm{o}}$ muertos & Mortalidad (\%) \\
\hline Hojas & 2500 & 10 & 0 & 0 \\
& 5000 & 10 & 1 & 10 \\
& 7500 & 10 & 2 & 20 \\
& 10000 & 10 & 3 & 30 \\
& 12500 & 10 & 7 & 70 \\
Jugo & 15000 & 10 & 9 & 90 \\
& 17500 & 10 & 10 & 100 \\
& 2500 & 10 & 0 & 0 \\
& 5000 & 10 & 0 & 0 \\
& 10000 & 10 & 1 & 10 \\
& 15000 & 10 & 2 & 20 \\
& 20000 & 10 & 4 & 40 \\
\hline
\end{tabular}

Tabla 3. Estimación de la dosis letal 50 (DL50) del extracto etanólico de las hojas y el jugo de P. Edulis

\begin{tabular}{lccc}
\hline Tratamiento & DL50 mg/kg & \multicolumn{2}{c}{ Límites de confianza 95\% } \\
\hline Hojas & 10687 & 9243 & 12203 \\
Jugo & 21471 & 17368 & 42769 \\
\hline
\end{tabular}

En la autopsia, no se encontró evidencias de alteraciones de los órganos, tales como hígado, bazo, riñones, corazón y pulmones. Tampoco hubo diferencias signifi- cativas entre los grupos, cuando se analizó el peso relativo de los órganos.

\section{DISCUSIÓN}

Los resultados muestran que, la presión arterial sistólica y la diastólica disminuyeron significativamente desde el primer día de tratamiento con el extracto etanólico de las hojas y el jugo del fruto de Passiflora edulis (maracuyá). Estudios previos han demostrado que otras plantas también tienen efecto antihipertensivo en modelos preclínicos y clínicos. Así, se ha evidenciado este efecto con los extractos de la hoja de olivo (Olea europaea), en animales de experimentación, tanto por vía oral como intravenosa $\left({ }^{21}\right)$. El ajo (Alium sativum) ha demostrado este efecto en estudios in vitro $\left.{ }^{(22}\right)$, en ratas hipertensas $\left({ }^{23}\right)$ y clínicamente en hipertensiones leves y moderadas $\left({ }^{24}\right)$. Otras plantas usadas en Colombia por la medicina folklórica para el tratamiento de hipertensión (Croton schiedeanus, Curatella americana y Lupinus amandus) también han demostrado tener efecto antihipertensivo en $\operatorname{ratas}\left({ }^{25}\right)$.

Dentro de los metabolitos secundarios de las plantas, los compuestos polifenólicos, especialmente los flavonoides, han demostrado tener efecto antihipertensivo en diferentes modelos experimentales. De esta manera, los polifenoles indujeron vasorrelajación dependiente del endotelio, a través del incremento de la producción de óxido nítrico en aorta de rata $\left({ }^{26-28}\right)$, así como en arteria coronaria porcina $\left({ }^{29}\right)$. Los flavonoides del jugo de uvas púrpuras incrementaron la liberación de óxido nítrico derivado de plaquetas y disminuyeron la producción de superóxido $\left({ }^{30}\right)$. Asimismo, se ha comunicado que el flavonoide luteolina incrementó la actividad del promotor de la sintasa del óxido nítrico endotelial y la expresión del ARNm de sintasa del óxido nítrico endotelial, con el 
consecuente incremento de óxido nítrico $\left({ }^{31}\right)$. Esto es relevante, en la medida que el óxido nítrico es el principal factor relajante derivado del endotelio, mientras que el factor hiperpolarizante derivado del endotelio también puede participar en la vasorrelajación, especialmente en pequeñas arterias, cuando está inhibida la formación de óxido nítrico. De esta manera, el óxido nítrico tiene un rol preponderante en la homeostasis de la presión sanguínea $\left({ }^{32}\right)$.

En modelos in vivo, utilizando ratas hipertensas, el flavonoide quercetina indujo una reducción significativa en la presión arterial sistólica, diastólica y media $\left({ }^{33}\right)$. Asimismo, los polifenoles exhibieron una disminución significativa de la presión sanguínea, explicada en parte por disminución de la expresión de cadenas ligeras de miosina fosforilada $\left({ }^{34}\right)$ y por incremento de la excreción urinaria de sodio, potasio y cloruro, además de aumentar la tasa de filtración glomerular $\left({ }^{35}\right)$.

Por otra parte, se ha informado que el eugenol produce relajación del músculo liso vascular, por bloqueo de canales de calcio sensibles a voltaje y los operados por receptor, que son modulados por óxido nítrico producido por el endotelio $\left.{ }^{36}\right)$.

La hipertensión arterial inducida por $\mathrm{N}_{\mathrm{G}}{ }^{-}$ nitro-L-arginina metil éster (L-NAME) no solo involucra la restricción de producción de óxido nítrico, sino también la activación del sistema nervioso simpático y la alteración del sistema renina-angiotensina, tal como incremento de la actividad de renina plasmática y de la concentración de angiotensina II o del número de receptores de angiotensina $\mathrm{I}\left({ }^{37}\right)$. En este modelo experimental, captopril, el fármaco de referencia, disminuyó la presión arterial de manera más efectiva que Passiflora. Este resultado confirma la eficiencia del tratamiento con inhibidores de la enzima convertidora de angiotensina para prevenir la hipertensión en ratas con deficiencia de óxido nítrico $\left({ }^{19}\right)$.
En ratas hipertensas se ha demostrado un incremento en la generación de anión superóxido en el endotelio vascular $\left({ }^{38}\right)$. Se cree que las especies reactivas del oxígeno contribuyen a la generación y/o mantenimiento de la hipertensión arterial por varios mecanismos, que incluyen: inactivación de óxido nítrico derivado del endotelio, generación no enzimática de isoprostanos $\mathrm{F}_{2}$ vasoconstrictores, por peroxidación de ácido araquidónico y depleción del cofactor de sintasa de óxido nítrico tetrahidrobiopterina $\left({ }^{39}\right)$. Los flavonoides tienen la propiedad de barrer radicales libres superóxido y además inhiben varias enzimas responsables de la síntesis de anión superóxido $\left({ }^{40}\right)$. Probablemente, los flavonoides de Passiflora contribuyen a su efecto antihipertensivo, además de su propiedad vasorrelajante, por su efecto antioxidante.

Es posible que los metabolitos secundarios presentes en las hojas y en el jugo del fruto de Passiflora edulis, principalmente flavonoides y eugenol, sean los responsables y expliquen en parte la actividad antihipertensiva demostrada en la presente investigación.

En relación al estudio de toxicidad aguda, se determinó que la dosis letal 50 (DL50) del extracto etanólico de las hojas de Passiflora edulis fue $10687 \mathrm{mg} / \mathrm{kg}$, mientras que del jugo del fruto fue aproximadamente el doble de aquél, lo cual permite clasificarlo como no tóxico (no clasificado, DL50 > $2000 \mathrm{mg} / \mathrm{kg}$ ), según las normas de la Comunidad Europea, para la clasificación de la toxicidad aguda oral $\left({ }^{41}\right)$.

En conclusión, en condiciones experimentales, el extracto etanólico de las hojas y el jugo del fruto de Passiflora edulis fueron efectivos en la disminución de la presión arterial en ratas hipertensas, y no presentan toxicidad aguda oral. Estos resultados podrían respaldar el empleo de esta planta en la medicina tradicional en pacientes hipertensos. 


\section{AGRADECIMIENTOS}

Al Consejo Superior de Investigaciones de la UNMSM, por la financiación del proyecto de investigación.

\section{REFERENCIAS BIBLIOGRÁFICAS}

1. Dhawan K, Dhawan S, Sharma A. Passiflora: a review update. Journal of Ethnopharmacology. 2004;94:1-23.

2. García M. Guía Técnica: Cultivo de maracuyá amarillo. San Andrés, El Salvador: Centro Nacional de Tecnología Agropecuaria y Forestal; 2002.

3. Centro de Capacitación y Experimentación 'Jesús Aguilar Paz'. El Cultivo de maracuyá (Passiflora Edulis Sims). La Fé, Cortes, Honduras: Centro Experimental; 2002.

4. Ministerio de Agricultura y Ganadería de Costa Rica. Aspectos Técnicos sobre Cuarenta y Cinco Cultivos Agrícolas de Costa Rica. San José, Sabana Sur,Costa Rica: Dirección General de Investigación y Extensión Agrícola; 1991.

5. Galindo F, Villavicencio M. Maracuyá. Seminario de Agronegocios. Lima: Universidad del Pacífico; 2000.

6. Jamir T, Sharma H, Dolui A. Folklore medicinal plants of Nagaland, India. Fitoterapia. 1999;70:395-401.

7. Desmarchelier C, Witting F. Setenta Plantas Medicinales de la Amazonía Peruana. $1^{\text {a }}$ ed. Lima: Gráfica Bellido; 2000. p. $187-90$.

8. Brack A. Diccionario enciclopédico de plantas útiles del Perú. $1^{\text {a }}$ Ed. Cusco: Centro de Estudios Regionales Andinos Bartolomé de las Casas; 1999. p. 368-70.

9. Natural Products Alert (NAPRALERT SM). Profile for Passiflora edulis. Chicago: The board of trustees of the University of Illinois; 2001.

10. Bombardelli E, Bonati A, Gabetta B, Martinelli E, Mustich G. Passiflorine, a new glycoside from Passiflora edulis. Phytochemistry. 1975; 14(12):2661-5.

11. Mareck U, Herrmann K, Galensa R, Wray V. The 6-Cchinovoside and 6-C-fucoside of luteolin from Passiflora edulis. Phytochemistry. 1991;30(10):3486-7.

12. Seigler D, Pauli G, Nahrstedt A, Leen R. Cyanogenic allosides and glucosides from Passiflora edulis and Carica papaya. Phytochemistry. 2002;60(8):873-82.

13. Christensen J, Jaroszewski J. Natural glycosides containing allopyranose from the passion fruit plant circular dichroism of benzaldehyde cyanohydrin glycosides. Org Lett. 2001;3(14):2193-5.

14. Slaytor M, McFarlane I. The biosynthesis and metabolism of harman in Passiflora edulis. Phytochemistry. 1968;7(4):605-11.

15. Yoshikawa K, Katsuta S, Mizumori J, Arihara S. Four cycloartane triterpenoids and six related saponins from Passiflora edulis. J Nat Prod. 2000;63(9):1229-34.

16. Bruschi M, Cardoso M, Milani H. Avaliaçao farmacológica de um extrato de Passiflora edulis variedade flavicarpa. Rev Ciênc Farm. 2002;23(2):263-76.
17. Petry R, Reginatto F, De Paris F, Gosmann G, Salgueiro J, Quevedo J, et al. Comparative pharmacological study of hydroethanol extracts of Passiflora alata and Passiflora edulis leaves. Phytother Res. 2001;15(2):162-4.

18. Puricelli L, Dell'Aica I, Sartor L, Garbisa S, Caniato R. Preliminary evaluation of inhibition of matrix-metalloprotease MMP-2 and MMP-9 by Passiflora edulis and P foetida aqueous extracts. Fitoterapia. 2003;74(3):302-4.

19. Pereira L, Bezerra D, Mandarim-de-Lacerda C. Aortic wall remodeling in rats with nitric oxide deficiency treated by enalapril or verapamil. Pathology Research and Practice. 2004;200:211-7.

20. Vega R, Carrillo C. Efecto sobre la motilidad intestinal y toxicidad aguda oral del extracto de Ocimun gratissimum $\mathrm{L}$. (orégano cimarrón). Rev Cubana Plant Med. 1997;2(2-3): 4-18.

21. Zarzuelo A, Duarte J, Jimenez J, González M, Utrilla M. Vasodilatador effect of olive leaf. Planta Med. 1991;57:4179.

22. Ganado P, Sanz M, Padilla E, Tejerina T. An in vitro study of different extracts and fractions of Allium sativum (garlic): vascular reactivity. Journal of Pharmacological Sciences. 2004;94:434-42.

23. Al-Qattan K, Khan I, Alnaqeeb M, Ali M. Mechanism of garlic (Allium sativum) induced reduction of hypertension in $2 \mathrm{~K}-1 \mathrm{C}$ rats: a possible mediation of $\mathrm{Na} / \mathrm{H}$ exchanger isoform1. Prostaglandins, Leukotrienes and Essential Fatty Acids. 2003;69:217-22.

24. Reuter H. Allium sativum and Allium ursinum: Part 2 Pharmacology and medical applications. Phytomedicine. 1995;2:73-91.

25. Guerrero M, Puebla P, Carron R, Martin M, Arteaga L, San Román L. Assessment of the antihypertensive and vasodilator effects of ethanolic extracts of some Colombian medicinal plants. J Ethnopharmacol. 2002;80:37-42.

26. Andriambelosom E, Kleschyov A, Muller B, Beretz A, Stoclet J, Andriantsitohaina R. Nitric oxide production and endotheliumdependent vasorelaxation induced by wine polyphenols in rat aorta. Brit J Pharmacology. 1997;120:1053-8.

27. Andriambelosom E, Magnier C, Haan-Archipoff G, Lobstein A, Anton R, Beretz A, Stoclet J, et al. Natural dietary polyphenolic compounds cause endothelium-dependent vasorelaxation in rat thoracic aorta. J Nutr. 1998;128:232433.

28. Benito S, Lopez D, Saiz M, Buxaderas S, Sánchez J, PuigParellada P, et al. A flavonoid-rich diet increases nitric oxide production in rat aorta. Brit J Pharmacology. 2002;135:910-6.

29. Taubert D, Berkels R, Klaus W, Roesen R. Nitric oxide formation and corresponding relaxation of porcine coronary arteries induced by plant phenols: Essential structural features. J Cardiovascular Pharmacology. 2002;40:701-13.

30. Freedman J, Parker C, Li L, Perlman J, Ivanov V, Deak L. Select flavonoids and whole juice from purple grapes inhibit platelet function and enhance nitric oxide release. Circulation. 2001;103:2792-8. 
31. Li H, Xia N, Brausch I, Yao Y, Forstermann U. Flavonoids from artichoke (Cynara scolymus L.) upregulate endothelialtype nitric-oxide synthase gene expression in human endothelial cells. J Pharmacol Experiment Ther. 2004;310(3):926-32.

32. Achike F, Kwan C. Nitric oxide, human diseases and the herbal products that affect the nitric oxide signalling pathway. Clinical and Experimental Pharmacology and Physiology. 2003;30:605-15.

33. Duarte J, Perez R, Vargas F, Angeles M, Perez F, Zarzuelo A, et al. Antihypertensive effects of the flavonoid quercetin in spontaneously hypertensive rats. Brit J Pharmacology. 2001;133:117-24.

34. Negishi H, Xi J, Ikeda K, Njelekela M, Nara Y, Yamori Y. Black and green tea polyphenols attenuate blood pressure increases in stroke-prone spontaneously hipertensive rats. J Nutr. 2004;134:38-42.

35. Jouad H, Lacaille-Dubois M, Lyoussi B, Eddouks M. Effects of the flavonoids extracted from Spergularia purpurea Pers. on arterial blood pressure and renal function in normal and hipertensive rats. J Ethnopharmacology. 2001;76:15963.

36. Nolf C, Venturini L, Valentim D. Vasorelaxant effects of eugenol on thoracic aorta. Vascular Pharmacology. 2003;40:59-66.

37. Bernátová I, Pechánova $\mathrm{O}$, Kristek F. Mechanism of structural remodelling of the rat aorta during long-term $\mathrm{N}_{\mathrm{G}}-$ nitro-L-arginina metil éster treatment. Jpn J Pharmacol. 1999;81:99-106.
38. Kerr S, Brosnan M, McIntire M, Reid J, Dominiczak A, Hamilton C. Superoxide anion production is increased in a model of genetic hipertensión: role of the endothelium. Hypertension. 1999;33(6):1353-8.

39. Vaziri N, Ni Z, Oveisi F, Trnavsky-Hobbs D. Effect of antioxidant therapy on blood pressure and NO synthase expression in hypertensive rats. Hypertension. 2000;36:95764.

40. Woodman O, Chan E. Vascular and anti-oxidant actions of flavonols and flavones. Clin Exp Pharmacol Physiol. 2004;31:786-90.

41. Unión Europea. Decisión 2000/368/CE de la Comisión: aproximación de las disposiciones legales, reglamentarias y administrativas en materia de clasificación, embalaje y etiquetado de las sustancias peligrosas. Diario Oficial. 2000;:L136:108-25.

Manuscrito recibido el 04 de setiembre de 2006 y aceptado para publicación el 29 setiembre de 2006.

\section{Correspondencia:}

\section{Juan Rojas Armas}

Instituto de Investigaciones Clínicas

Hospital Nacional Dos de Mayo

Av. Grau cuadra 13. Parque Historia de la Medicina.

Lima 1, Perú.

Correo-e: jprojasarmas@yahoo.com 